\title{
Contribuição de Melampus coffeus (Gastropoda, Ellobiidae) na degradação da serapilheira do médio estuário do rio Pacoti, Ceará, Brasil
}

\author{
Diva S. Tavares ${ }^{1}$, Rafaela C. Maia ${ }^{2}$ \& Cristina A. Rocha-Barreira ${ }^{3}$
}

\begin{abstract}
1. Programa de Pós-Graduação em Ecologia e Recursos Naturais, Departamento de Biologia, Centro de Ciências, Universidade Federal do Ceará, Campus do Pici, Fortaleza, CE, Brasil. (divast@hotmail.com)

2. Instituto Federal de Educação, Ciência e Tecnologia do Ceará, Campus Acaraú. (rafaelacmaia@yahoo.com.br)

3. Laboratório de Zoobentos, Instituto de Ciências do Mar, Universidade Federal do Ceará, Av. Abolição, 3207, 60165-081 Fortaleza, CE, Brasil. (cristina.labomar@gmail.com)
\end{abstract}

\begin{abstract}
Contribution of Melampus coffeus (Gastropoda, Ellobiidae) to leaf litter decomposition in the middle estuary of the Pacoti river, Ceará, Brazil. The leaf litter decomposition in the middle estuary of the Pacoti river was studied in the presence and absence of the gastropod Melampus coffeus (Linnaeus, 1758), aiming at examining the contribution of this invertebrate to the organic matter cycling in mangroves ecosystems. The local population density of $M$. coffeus and leaf distribution based on wet biomass, dry biomass, and abundance, were estimated. A field experiment was conducted using samples of Rhizophora mangle (Rhizophoraceae) leaves (with similar color, and wet and dry biomass) equally distributed in 64 leaf litter cages. Groups of 14 individuals of $M$. coffeus were placed in 32 of these cages. The experimental cages were equally distributed with their respective control cages (leaves only) in the field among four points, totaling 64 cages. During eight weeks four pairs of cages (experimental and control) were collected weekly. Once collected, the leaves showing signs of grazing by $M$. coffeus were counted. The wet and dry biomasses of samples were also determined. Although $M$. coffeus can contribute to leaf litter degradation, in this study, its effect on leaf degradation was not significant when comparing experiment and control groups during the experimental time in the studied area.
\end{abstract}

KEYWORDS. Leaves, macrodetritivores, mangroove, Rhizophora mangle.

RESUMO. A degradação da serapilheira no médio estuário do rio Pacoti foi estudada na presença e na ausência do gastrópode Melampus coffeus (Linnaeus, 1758), visando verificar a possível contribuição desse invertebrado na ciclagem da matéria orgânica dos manguezais. A densidade populacional de $M$. coffeus e a distribuição das folhas, em termos de biomassa úmida, biomassa seca e abundância, presentes na serapilheira do local, foram estimadas a partir de uma amostragem. Um experimento em campo foi realizado com amostras de coloração e biomassas similares de folhas de Rhizophora mangle (Rhizophoraceae) sem sinais de herbivoria, distribuídas igualmente entre 64 gaiolas. Em 32 destas foram colocados também exemplares de M. coffeus. Essas gaiolas foram distribuídas entre quatro pontos, sendo oito pares de gaiolas (controle e experimento) por ponto. Durante oito semanas, quatro pares de gaiolas foram coletados semanalmente. Após coletadas, os sinais de pastagem nas folhas causados por $M$. coffeus foram contados e as biomassas úmida e seca das amostras foram determinadas. Comparando controle e experimento, verificou-se que M. coffeus é capaz de contribuir na degradação da serapilheira na área estudada, porém, não foi verificada uma participação significativa desse gastrópode na degradação das folhas quando comparados experimento e controle ao longo do experimento.

PALAVRAS-CHAVE. Folhas, macrodetritívoros, manguezal, Rhizophora mangle.

As folhas da serapilheira apresentam uma carga significativa de matéria orgânica que se torna disponível com os processos de decomposição, fornecendo uma importante fonte de carbono, nitrogênio e fósforo que flui dos manguezais para outros ecossistemas aquáticos (AkÉ-CASTILLO et al., 2006). A macrofauna pode acelerar o processo de degradação da serapilheira pelo consumo direto ou pela segmentação das folhas recém-caídas no solo (ROBERTSON \& DANIEL, 1989).

Devido à elevada densidade e ao fato de ocuparem uma posição trófica entre microorganismos decompositores e populações de consumidores maiores, os gastrópodes, como os do gênero Melampus Montfort, 1810, são potencialmente importantes nos processos de mineralização e transferência de energia dentro dos campos alagados (Graça et al., 2000).

A família Ellobiidae constitui um grupo de moluscos pulmonados primitivos. Em sua maioria, são halofílicos e vivem nas zonas superiores entremarés, sendo comumente encontrados nos manguezais (MARTINS, 1996) e em praias pedregosas (PAREDEs et al., 2005). Moluscos do gênero Melampus respiram o ar através do teto da cavidade do manto vascularizada que funciona como pulmão, mas dependem da água para a reprodução (RUSSEL-HunTer et al., 1972).

Melampus coffeus (Linnaeus, 1758) é comum nos manguezais e marismas do Oceano Atlântico, com distribuição da Flórida ao Uruguai (RIos, 1994). Os indivíduos considerados adultos são facilmente encontrados na serapilheira. Segundo Proffitt et al. (1993) e Proffitt \& Delvin (2005), M. coffeus alimenta-se de detritos vegetais, consumindo folhas do mangue e, portanto, assume papel importante nesse ecossistema.

A maioria dos trabalhos publicados sobre esses animais foram realizados na América do Norte, notadamente na Flórida (Моoк, 1986; ProfritT et al., 1993; Proffitt \& Delvin, 2005). No Brasil, até o momento, o único trabalho publicado sobre esse gastrópode foi realizado no estado do Ceará, na praia de Arpoeiras, região de Acaraú, por MAIA \& TANAKA (2007). Os autores avaliaram os efeitos das espécies de mangue na distribuição espacial de $M$. coffeus em relação à densidade 
de indivíduos $/ \mathrm{m}^{2}$ e ao tamanho da concha e verificaram que árvores de diferentes espécies no mangue podem servir de substrato para esses animais durante as marés cheias, mas há uma divisão de tamanho dos moluscos de acordo com o substrato disponível.

Com o presente trabalho, objetivou-se verificar a participação do gastrópode $M$. coffeus na degradação das folhas da serapilheira no médio estuário do rio Pacoti, Ceará, levando em consideração a densidade média e a distribuição das folhas da serapilheira, quanto à biomassa úmida e biomassa seca no local e, assim, contribuir para o conhecimento sobre essa espécie tão comum em manguezais, porém ainda pouco estudada.

\section{MATERIAL E MÉTODOS}

Área de estudo. O estudo foi realizado no médio estuário do rio Pacoti, no município de Eusébio, Ceará, Brasil (0349'59,2' S, 38²5'13,0', W), entre setembro e novembro de 2007. A região apresenta clima tropical quente subúmido, com temperatura média variando de 26 a $28^{\circ} \mathrm{C}$ e pluviosidade aproximada de $1379,9 \mathrm{~mm}$ por ano, sendo o período chuvoso de janeiro a maio (IPECE, 2009).

O rio Pacoti é o maior dos cursos d’água que atravessam a região metropolitana de Fortaleza, estando sua nascente na vertente-oriental da serra de Baturité, percorrendo $150 \mathrm{~km}$ até desembocar no mar (OLIVEIRA et al., 1988). A vegetação mais marcante ao longo das margens do estuário é a floresta de mangue, denominada de floresta Perenifólia Paludosa Marítima, que se alonga cerca de $15 \mathrm{~km}$ a partir da foz do rio, até as proximidades da cidade de Aquiraz. Tal floresta é composta, principalmente pelas espécies Rhizophora mangle (Rhizophoraceae), Laguncularia racemosa (Combretaceae), Avicennia sp. (Avicenniaceae) e Conocarpus erecta (Combretaceae).

Métodos. A fim de estimar a quantidade de folhas e de $M$. coffeus presentes na serapilheira do local, foi realizada uma coleta piloto em uma área de franja dominada por $R$. mangle. Tal coleta se deu em quatro pontos separados por uma distância mínima de $10 \mathrm{~m}$ um do outro. Os pontos de coleta foram escolhidos aleatoriamente na margem esquerda do rio Pacoti, tendo como base o Centro de Estudos Ambientais e Costeiros do Instituto de Ciências do Mar.

Com o auxílio de um quadrado de 25 x $25 \mathrm{~cm}$, foram coletadas ao acaso cinco amostras de serapilheira de cada ponto. De cada área marcada pelo quadrado, foram analisados os exemplares de $M$. coffeus, sendo estes contados e medidos do ápice à base da concha com paquímetro de precisão de $0,05 \mathrm{~mm}$. No laboratório, as amostras de serapilheira foram processadas, contadas e pesadas. As folhas foram colocadas em estufa a $70^{\circ} \mathrm{C}$ até obtenção de massa constante ( $\mathrm{g}$ de massa seca).

Para avaliar a contribuição de $M$. coffeus na degradação da serapilheira no local em estudo, foram confeccionadas 64 gaiolas com dimensões de 25 x 25 x 5 $\mathrm{cm}$, em forma de paralelepípedo, sendo as arestas constituídas de arame galvanizado e as faces por telas de nylon com abertura de $1 \mathrm{~mm}^{2}$.

A partir da média geral de biomassa úmida de folhas encontradas na serapilheira na amostragem piloto, foram coletadas folhas inteiras, de tamanhos similares, de cor amarela e sem sinais de herbívoria entre as raízes de Rhizophora mangle, as quais foram distribuídas em 68 amostras, com biomassa úmida variando de 22 a $23 \mathrm{~g}$. Sessenta e quatro destas foram colocadas nas gaiolas (uma amostra por gaiola). As quatro restantes foram submetidas à secagem em estufa a $70^{\circ} \mathrm{C}$ até obter massa constante ( $\mathrm{g}$ de peso seco) para serem utilizadas como referência de biomassa seca inicial das amostras utilizadas no experimento.

Com base nos valores das médias gerais do tamanho da concha e do número de indivíduos de Melampus coffeus encontrados na amostragem piloto foi realizada uma coleta de 448 exemplares de M. coffeus (com comprimento da base ao ápice da concha variando de 12 a $18 \mathrm{~mm}$ ) para serem utilizados no experimento. Os animais foram mantidos em laboratório (sob temperatura ambiente e condições normais de claro e escuro) dentro de recipientes plásticos fechados com tela de nylon (abertura $1 \mathrm{~mm}^{2}$ ) e submetidos a jejum por 48 horas. Após esse período, foram distribuídos em 32 das 64 gaiolas utilizadas no experimento de modo que cada gaiola recebeu um grupo de 14 exemplares. Em seguida, as gaiolas foram fechadas por costura e levadas para o campo, sendo 32 gaiolas com $M$. coffeus e folhas (experimento) e 32 contendo apenas folhas (controle).

Em cada ponto escolhido foram colocadas 16 gaiolas, sendo oito gaiolas experimento e oito controle. Todas as gaiolas foram devidamente etiquetadas e amarradas com cordas aos pares (experimento e controle) às raízes de $R$. mangle. Os pares foram distribuídos aleatoriamente entre os quatro pontos escolhidos inicialmente (os mesmos da coleta piloto) de modo que a distância entre cada par foi de 0,5 a 3,5 m.

Semanalmente de setembro a novembro de 2007 foram retirados do campo quatro pares de gaiolas, sendo um par de cada ponto. Após coletadas, as gaiolas foram abertas e as folhas retiradas, limpas com papel toalha para retirar o sedimento incrustado e analisadas para identificar sinais de herbivoria causados por $M$. coffeus.

O cálculo da medida da área foliar, apesar de permitir análises mais precisas sobre a atuação desses gastrópodes na degradação das folhas de mangue (Proffitt et al., 1993; Proffitt \& Delvin, 2005), não foi utilizada no presente trabalho em virtude das condições as quais as folhas foram submetidas. Algumas delas ficaram danificadas possivelmente pela ação da variação diária das marés e do acumulo de sedimento. Dessa forma, a medida da área foliar não seria viável para todas as folhas. Assim, aquelas com sinais de pastagem causados por $M$. coffeus foram apenas contadas com o intuito de avaliar se o número de folhas pastadas aumentava, diminuía ou não sofria alteração significativa ao longo do tempo de experimentação. Após a análise visual, foi verificada a biomassa úmida das folhas e, em seguida, as mesmas foram colocadas em estufa a $70^{\circ} \mathrm{C}$ até obtenção de massa constante.

Todos os dados obtidos na amostragem-piloto e no experimento de campo foram testados quanto a sua normalidade e homogeneidade de variância e, quando necessário, sofreram transformação do tipo $\mathrm{y}=\log (\mathrm{x}+1)$. As comparações entre as médias foram realizadas através 
da análise de variância paramétrica (ANOVA). Para a definição do peso seco inicial estimado das folhas colocadas nas gaiolas do experimento, foi realizada análise de regressão de Sperman. Todas as análises foram realizadas através do software STATISTICA versão 6.0 (STAT Soft InCORPORATION, 2003).

\section{RESULTADOS}

A média da massa úmida das amostras de folhas não apresentou diferença significativa entre os quatro pontos $\left(\mathrm{F}_{1,3}=2,8390 ; \mathrm{p}=0,070\right)$. No entanto, a média da massa seca $\left(\mathrm{F}_{1,3}=4,6983 ; \mathrm{p}=0,015\right)$ e do número de folhas $\left(\mathrm{F}_{1,3}=5,647 ; \mathrm{p}=0,007\right)$ (valores transformados) presentes nas vinte amostras diferiram significativamente (Tab. I). Neste último aspecto, verificou-se uma quantidade de folhas mais elevada no ponto 1 em relação aos demais. As folhas das amostras apresentaram aspectos bastante diversificados, incluindo diferentes estágios de senescência e decomposição, portanto com diferentes colorações, além de uma grande variedade dimensional.

$\mathrm{Na}$ amostragem, foram coletados 136 exemplares de Melampus coffeus, sendo $37\left(118,4\right.$ ind./ $\left./ \mathrm{m}^{2}\right)$ encontrados no primeiro ponto, $36\left(115,2 \mathrm{ind} . / \mathrm{m}^{2}\right)$ no segundo, $26(83,2$ ind. $\left./ \mathrm{m}^{2}\right)$ no terceiro e $37\left(118,4 \mathrm{ind} . / \mathrm{m}^{2}\right)$ no quarto ponto (Tab. II). Não houve diferença significativa entre a abundância de $M$. coffeus encontrada em cada ponto de coleta $\left(\mathrm{F}_{1,3}=0,25924 ; \mathrm{p}=0,85\right)$, tendo sido coletados, em média entre 5,2 e 7,4 exemplares por amostra. A densidade média desses animais na área em estudo foi de 108,8 ind./ $\mathrm{m}^{2}$ (Tab. II). O tamanho da concha dos indivíduos coletados variou de 4,5 a 17,5 mm, havendo diferença significativa quanto ao tamanho médio dos animais entre os pontos de coleta $\left(\mathrm{F}_{1,3}=30,254 ; \mathrm{p}<0,001\right)$, sendo os menores encontrados no ponto 1 (Tab. II). Portanto, os resultados da amostragem piloto indicaram que na área estudada a população de $M$. coffeus se encontra, quantitativamente, distribuída de forma relativamente homogênea entre os quatro pontos. Porém, tal padrão não foi verificado quanto ao tamanho da concha dos animais. Neste caso, os menores indivíduos foram encontrados no primeiro ponto.

Considerando os valores de $\mathrm{p}$ das médias por amostra das massas úmida e seca e da abundância das folhas coletadas foi utilizado o dobro da média geral de massa úmida das folhas observada na amostragem para padronizar as amostras que foram colocadas nas gaiolas. Assim, em cada gaiola foi colocada uma amostra com massa úmida variando de 22 a 23 g. A análise de regressão entre a biomassa úmida e a biomassa seca das quatro amostras de folhas amarelas de $R$. mangle indicou haver correlação entre essas biomassas $\left(\mathrm{R}^{2}=0,7788\right)$, fornecendo assim um resultado consistente para a estimativa da biomassa seca inicial das amostras de folhas colocadas em campo no interior das gaiolas (Fig. 1).

Também com base nos dados da amostragem piloto foi definido o número de animais que foram colocados em cada gaiola do experimento, de modo que o valor

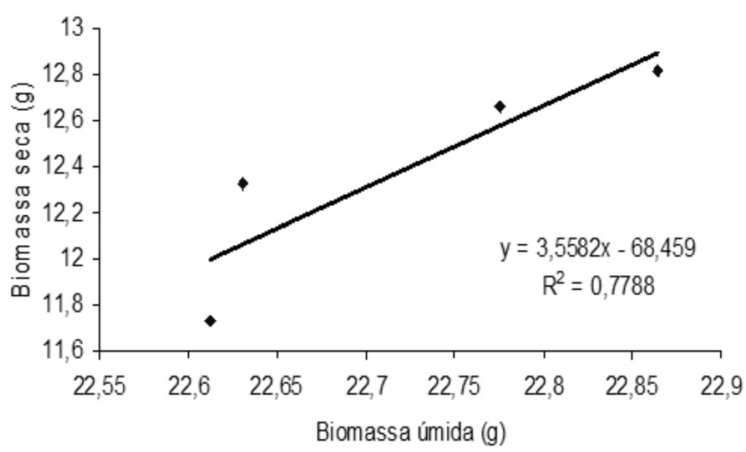

Figura 1. Análise de regressão de Sperman obtida a partir das amostras de folhas utilizadas como referência para o cálculo da biomassa seca inicial das amostras submetidas ao experimento de campo, na margem do rio Pacoti, Ceará, Brasil.

Tabela I. Médias por amostra das massas (em gramas) úmida e seca e da abundância das folhas coletadas na serapilheira na margem do médio estuário do rio Pacoti, Ceará, Brasil.

\begin{tabular}{|c|c|c|c|c|c|c|}
\hline \multirow[t]{2}{*}{ Pontos } & \multicolumn{2}{|c|}{ Massa úmida } & \multicolumn{2}{|c|}{ Massa seca } & \multicolumn{2}{|c|}{ Número de folhas } \\
\hline & Média (g) & Desvio padrão & Média $(\mathrm{g})$ & Desvio padrão & Média & Desvio padrão \\
\hline 1 & 18,03 & 10,56 & 6,99 & 3,14 & 20,20 & 10,35 \\
\hline 2 & 5,28 & 2,93 & 2,12 & 1,25 & 4,80 & 1,79 \\
\hline 3 & 11,99 & 6,74 & 4,13 & 2,33 & 10,00 & 5,70 \\
\hline 4 & 10,12 & 5,49 & 3,38 & 1,12 & 8,60 & 4,88 \\
\hline Média geral & 11,35 & 6,43 & 4,15 & 1,96 & 10,90 & 5,68 \\
\hline $\mathrm{p}$ & \multicolumn{2}{|c|}{0,070} & \multicolumn{2}{|c|}{0,015} & \multicolumn{2}{|c|}{0,007} \\
\hline
\end{tabular}

Tabela II. Médias do número de indivíduos por amostra, tamanho da concha e densidade de Melampus coffeus (Linnaeus, 1758) coletados na margem do médio estuário do rio Pacoti, Ceará, Brasil.

\begin{tabular}{lcccccc}
\hline Pontos & \multicolumn{2}{c}{ Tamanho da concha } & \multicolumn{2}{c}{ Número de indivíduos } & \multicolumn{2}{c}{ Densidade } \\
\cline { 2 - 7 } & Média $(\mathrm{mm})$ & Desvio padrão & Média & Desvio padrão & Média (indivíduos/m²) & Desvio padrão \\
\hline 1 & 11,18 & 2,33 & 7,40 & 3,57 & 118,40 & 57,24 \\
2 & 15,01 & 1,28 & 7,20 & 1,30 & 115,20 & 20,86 \\
3 & 14,28 & 1,77 & 5,20 & 1,92 & 83,20 & 30,77 \\
4 & 14,18 & 1,94 & 7,40 & 4,83 & 118,40 & 77,23 \\
Média geral & 13,60 & 2,40 & 6,80 & 3,10 & 108,80 & 49,68 \\
p & & & & 0,73 & & \\
\end{tabular}


duplicado da média geral do número de indivíduos foi utilizado. Assim, o valor médio de 6,8 indivíduos por amostra foi considerado igual a sete e este valor duplicado (14 exemplares por gaiola). Para o tamanho da concha foi utilizada a média como referência, sendo que foram utilizados apenas animais com concha de tamanho igual ou superior a $12 \mathrm{~mm}$.

Não foi verificada diferença significativa no número de folhas consumidas ao longo do tempo entre as gaiolas do experimento $\left(\mathrm{F}_{1,48}=1,256 ; \mathrm{p}=0,2680\right)$ (Fig. 2). Em relação à biomassa úmida das folhas (Fig. 3), houve um aumento considerável a partir da quinta semana. No mesmo período, foram observadas algumas alterações no campo, como a presença de um biofilme sobre o sedimento e que se acumulou também sobre as gaiolas e as folhas em seu interior. No decorrer do experimento, observou-se diminuição da biomassa seca (Fig. 4) perdida das folhas das gaiolas com M. coffeus. Entretanto, esta diminuição não foi estatisticamente significativa $\left(\mathrm{F}_{1,48}=1,251 ; \mathrm{p}=0,20\right)$.

As biomassas úmidas e secas finais apresentaram diferenças significativas ao longo do tempo $\left(\mathrm{F}_{1,7}=7,312\right.$; $\mathrm{p}=0,0003$ e $\mathrm{F}_{1,7}=2,895 ; \mathrm{p}=0,0117$ respectivamente). Entretanto, estas diferenças foram observadas sem distinção entre as gaiolas do experimento e do controle.

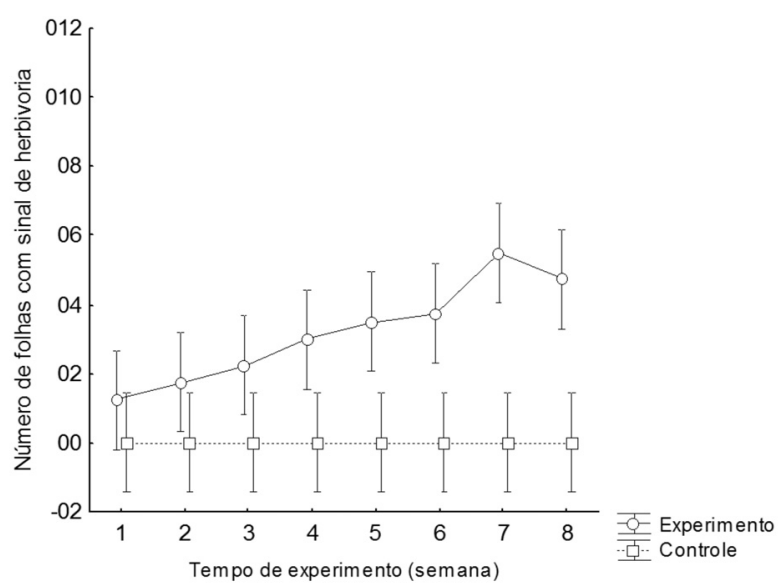

Figura 2. Número médio $( \pm \mathrm{EP})$ de folhas pastadas por Melampus coffeus (Linnaeus, 1758) ao final de cada semana na margem do rio Pacoti, Ceará, Brasil.

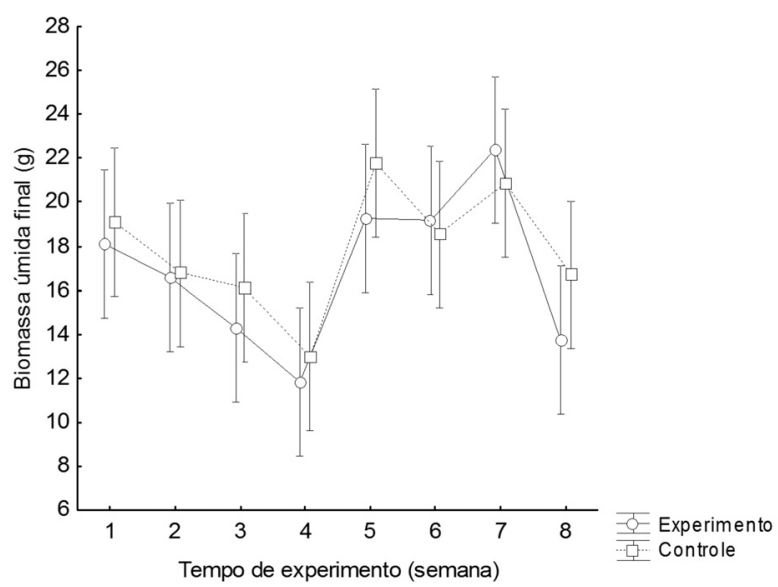

Figura 3. Comparação entre as biomassas finais úmidas $( \pm \mathrm{EP})$ das folhas das amostras das gaiolas controle e experimento a cada semana na margem do rio Pacoti, Ceará, Brasil.

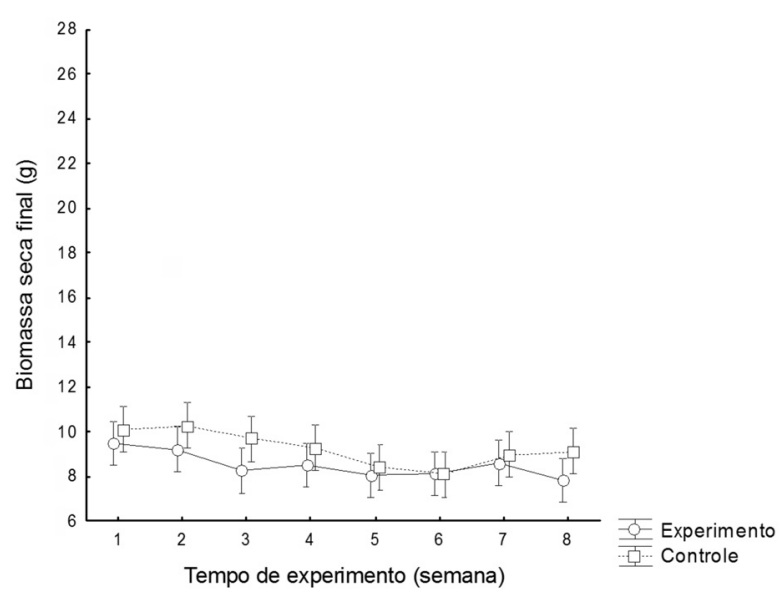

Figura 4. Comparação entre as biomassas finais secas ( $\pm \mathrm{EP})$ entre as amostras de folhas das gaiolas controle e experimento a cada semana na margem do rio Pacoti, Ceará, Brasil.

\section{DISCUSSÃO}

A seleção da área para a amostragem foi realizada considerando a semelhança entre os pontos de coleta, levando-se em conta a distância da borda do leito estuarino, o início da área vegetada e a equidistância entre os pontos. Entretanto, os resultados obtidos quanto a biomassa seca e o número de folhas encontradas na serapilheira por $\mathrm{m}^{2}$, apontaram que no ponto 1 as condições ambientais são provavelmente diferentes dos outros três pontos. É possível que o arranjo estrutural dos troncos, galhos e raízes das árvores promovam uma maior retenção de folhas, podendo acarretar aumento da umidade. Esta condição diferenciada poderia, por exemplo, favorecer a predominância de indivíduos de menor tamanho, possivelmente mais sensíveis à dessecação.

Considerando a íntima relação que se estabelece entre os seres vivos e o material abiótico que constituem os manguezais, a densidade populacional é certamente um fator importante. ProffitT et al. (1993) verificaram em Boca Ciega, Flórida densidades de até 130,2 M. coffeus/ $\mathrm{m}^{2}$. Também em Boca Ciega Proffitt \& Delvin (2005) verificaram uma densidade média de 142,7 M. coffeus $/ \mathrm{m}^{2}$ após quase três anos de observações. Portanto, a densidade populacional de $M$. coffeus observada no presente estudo (considerando apenas os valores absolutos) é similar àquelas encontradas nos estudos realizados na Flórida, indicando que a espécie pode ter distribuição e abundância similar nos manguezais do continente americano.

Melampus coffeus é, portanto, um macrodetritívoro abundante em manguezais, alimentando-se preferencialmente de folhas de mangue caídas. Outrossim, demonstra preferência por certas espécies (e.g. Rhizophora mangle e Avicennia germinans) e consome mais rapidamente folhas mais velhas (PROFFITT et al., 1993; ProffitT \& Devlin, 2005).

ProffitT et al. (1993) realizaram um experimento em campo semelhante a este para verificar a taxa de consumo do $M$. coffeus em folhas de A. germinans, $R$. mangle e Laguncularia racemosa. Aqueles autores constataram que não houve diferença significativa em relação ao 
controle nos dez primeiros dias de exposição das folhas ao gastrópode, mas a perda da área foliar das folhas expostas ao $M$. coffeus depois desse período foi sempre maior do que nas folhas controle até o $53^{\circ}$ dia do experimento. FARNSWORTH \& ELLISON (1993), analisando o impacto causado por herbívoros em folhas de $R$. mangle, observaram os primeiros sinais de consumo ainda na primeira semana. No presente trabalho, os sinais de pastagem causados por $M$. coffeus foram percebidos logo após a primeira semana de experimento, mas esse consumo não se refletiu em uma redução significativa da biomassa quando comparada ao controle.

O aumento da biomassa úmida e seca das folhas observado a partir da quinta semana de experimento pode ter sido provocado pelo acúmulo de biofilme sobre as folhas que surgiu na área em estudo de forma abundante a partir da quarta semana de experimento. Apesar de não ter sido realizada qualquer análise que pudesse comprovar a composição do biofilme, é possível que o mesmo tenha sido formado a partir da proliferação de organismos como fungos e cianobactérias. Segundo Coelho et al. (2004), cianobactérias (encontradas em forma de filamentos vermelhos, verdes ou quase negros sobre mangues, solos e algas), fungos e muitos protistas (e.g. diatomáceas) podem ser encontrados associados ao ecossistema manguezal, vivendo nos sedimentos associados às plantas.

As folhas utilizadas nas gaiolas do experimento não sofreram consumo significativo (biomassa seca perdida) pela ação de pastagem do $M$. coffeus durante o período de experimentação. Porém, as condições experimentais podem ter promovido uma situação de estresse para os animais e ter influenciado no comportamento alimentar destes. Por outro lado, o tempo de duração do experimento pode não ter sido longo o bastante para que fosse possível constatar alguma tendência no aumento da taxa de degradação das folhas expostas. Também é possível que o número de réplicas não tenha sido suficiente para confirmar que o $M$. coffeus é capaz de contribuir efetivamente na degradação da serapilheira da área estudada.

Certamente esses fatos representam um estímulo para a realização de novos e mais longos experimentos incluindo maior número de repetições e em diferentes épocas do ano, considerando os períodos de seca e chuva da região. Além disso, a realização de estudos em outros manguezais forneceria informações a respeito das relações ecológicas existentes nesses ecossistemas e permitindo comparar se a taxa de consumo de folhas de serapilheira por $M$. coffeus entre diferentes manguezais (de diferentes condições físicas e composição florística).
Agradecimentos. Ao Vitor Luz, pela colaboração durante as coletas no campo e ao Centro de Estudos Ambientais e Costeiros do Labomar, por facilitar nosso acesso à área de estudo.

\section{REFERÊNCIAS BIBLIOGRÁFICAS}

Aké-Castillo, J. A; Vazquez, G. \& López-Portillo, J. 2006. Litterfall and decomposition of Rhizophora mangle L. in a coastal lagoon in the southern Gulf of Mexico. Hydrobiologia 559(1): $101-111$

Coelho, P. A.; Batista-Leite, L. M. A.; dos Santos, M. A. C. \& Torres, M. F. A. 2004. O manguezal. In: EskinaZi-LeÇA, E.; Neumann-Leitão, S. \& DA Costa, M. F. eds. Oceanografia: Um cenário tropical. Recife, Bagaço. p.640-688.

Farnsworth, E. J. \& Ellison, A. M. 1993. Dynamics of herbivory in Belizean mangal. Journal of Tropical Ecology 9(4):435453

GraçA, M. A.; Newell, S. Y. \& Kneib, R. T. 2000. Grazing rates of organic matter and living fungal biomass of decaying Spartina alterniora by three species of salt-marsh invertebrates. Marine Biology 136:281-289.

IPECE. (Instituto de Pesquisa e Estratégia Econômica do Ceará). 2009. Perfil básico Municipal - Eusébio. Fortaleza, Governo de Estado do Ceará. 17p.

MaiA, R. C. \& TAnaKA, M. O. 2007. Avaliação dos efeitos de espécies de mangue na distribuição de Melampus coffeus (Gastropoda, Ellobidae) no Ceará, nordeste do Brasil. Iheringia, Série Zoologia, 97(4):379-382.

Martins, A. M. F. 1996. Origen and evolutionary radiation of the Mollusca: relationships within the Ellobiidae Oxford, Oxford University. 392p.

Mook, D. 1986. Absorption efficiencies of the intertidal mollusk Acanthopleura granulata Gmelin. Marine Ecology 7:105-113.

Oliveira, A. M. E.; Irving, M. A. \& Lima, H. H. 1988. Aspectos bioecológicos do estuário do rio Pacoti, Ceará, Brasil. Arquivos de Ciências do Mar 27:91-100.

Paredes, C.; Indacochea, A.; Cardoso, F. \& Ortega, K. 2005. Familia Ellobiidae (Gastropoda: Archaeopulmonata) en el litoral peruano. Revista Peruana de Biología 12(1):69-76.

ProffitT, C. E. \& Devlin, D. J. 2005. Grazing by the intertidal gastropod Melampus coffeus greatly increases mangrove leaf litter degradation rates. Marine Ecology Progress Series 296:209-218

Proffitt, C. E.; Johns, K. M.; Cochrane C. B.; Devlin, D. J.; Reynolds, T. A.; Payne, D. L.; Jeppsen, S.; Peel, D. W. \& Linden, D. 1993. Field and laboratory experiments on the consumption of mangrove leaf litter by the macrodetritivore Melampus coffeus L. (Gastropoda: Pulmonata). Biological Sciences 56(4):211-222.

Rios, E. C. 1994. Seashells of Brazil. 2.ed. Rio Grande, Museu Oceanográfico da FURG. 368p.

Robertson, A. I. \& Daniel, P. A. 1989. Decomposition and the Annual Flux of Detritus from Fallen Timber in Tropical Mangrove Forests. Limnology and Oceanography 34(3):640-646.

Russel-Hunter, W. D.; Apley, M. L. \& Hunter, R. D. 1972. Earlylife-history of Melampus and the significance of semilunar synchrony. Biology Bulletin 143:625-656.

Stat Soft Incorporation. 2003. Statistica. Sistema de software para análise de dados. Disponível em: <http:// www.statsoft.com.br/pt/index.php>. Acesso em: 01.12.2010. 